\title{
REVIEW
}

\section{Review of Hybrid (Deterministic/Monte Carlo) Radiation Transport Methods, Codes, and Applications at Oak Ridge National Laboratory*}

\author{
John C. WAGNER ${ }^{* *}$, Douglas E. PEPLOW, Scott W. MOSHER and Thomas M. EVANS
}

Oak Ridge National Laboratory, Oak Ridge, TN 37831, USA

\begin{abstract}
This paper provides a review of the hybrid (Monte Carlo/deterministic) radiation transport methods and codes used at the Oak Ridge National Laboratory and examples of their application for increasing the efficiency of real-world, fixed-source Monte Carlo analyses. The two principal hybrid methods are (1) Consistent Adjoint Driven Importance Sampling (CADIS) for optimization of a localized detector (tally) region (e.g., flux, dose, or reaction rate at a particular location) and (2) Forward Weighted CADIS (FW-CADIS) for optimizing distributions (e.g., mesh tallies over all or part of the problem space) or multiple localized detector regions (e.g., simultaneous optimization of two or more localized tally regions). The two methods have been implemented and automated in both the MAVRIC sequence of SCALE 6 and ADVANTG, a code that works with the MCNP code. As implemented, the methods utilize the results of approximate, fast-running 3-D discrete ordinates transport calculations (with the Denovo code) to generate consistent space- and energy-dependent source and transport (weight windows) biasing parameters. These methods and codes have been applied to many relevant and challenging problems, including calculations of PWR ex-core thermal detector response, dose rates throughout an entire PWR facility, site boundary dose from arrays of commercial spent fuel storage casks, radiation fields for criticality accident alarm system placement, and detector response for special nuclear material detection scenarios and nuclear well-logging tools. Substantial computational speed-ups, generally $O\left(10^{2-4}\right)$, have been realized for all applications to date. This paper provides a brief review of the methods, their implementation, results of their application, and current development activities, as well as a considerable list of references for readers seeking more information about the methods and/or their applications.
\end{abstract}

KEYWORDS: Monte Carlo, variance reduction, hybrid methods, CADIS, FW-CADIS

\section{Introduction}

In the field of nuclear engineering, deterministic (e.g., discrete ordinates) and stochastic (Monte Carlo) methods are widely used to solve radiation transport problems. Each method has strengths and weaknesses. Deterministic methods produce detailed, system-wide solutions and are computationally efficient. However, deterministic methods contain uncertainties associated with the discretization of the independent variables (space, energy and angle) of the transport equation and can admit solutions that exhibit non-physical features (e.g., ray effects and negative fluxes). This is especially the case in shielding applications. Hence, a significant degree of insight and expertise is required to mitigate these undesirable characteristics and ultimately produce usable results. The Monte Carlo (MC) method enables detailed, explicit geometric, energy, and angular representations and hence is considered the most accurate method available for solving complex radiation transport problems. However, due

\footnotetext{
*Notice: This manuscript has been authored by UT-Battelle, LLC, under contract DE-AC05-00OR22725 with the U.S. Department of Energy. The United States Government retains and the publisher, by accepting the article for publication, acknowledges that the United States Government retains a non-exclusive, paid-up, irrevocable, world-wide license to publish or reproduce the published form of this manuscript, or allow others to do so, for United States Government purposes.

**Corresponding author, E-mail: wagnerjc@ornl.gov
}

to its nature of simulating individual particles and inferring the average behavior of the particles in the system from the average behavior of the individually simulated particles, the MC method is very computationally intensive, especially for deep-penetration type problems. Despite substantial advancements in computational hardware performance and widespread availability of parallel computers, the computer time required for analog $\mathrm{MC}$ is still considered exorbitant and/or prohibitive for the design and analysis of many relevant real-world nuclear applications.

For such applications, variance reduction (VR or biasing) techniques are required to enable the MC calculation of the quantities of interest with the desired statistical uncertainty and the available computational resources. The main difficulty associated with using variance reduction techniques is the determination of the problem-dependent parameters required by the variance reduction techniques. In the absence of a reliable, automated process, determination of effective variance reduction parameters is an iterative, manual process that, depending on the problem difficulty, can require considerable time, expertise, and experience. Responding to this difficulty, a number of strategies (both deterministic and stochastic) for automated determination of variance reduction parameters have been proposed and developed. A review of variance reduction concepts and previous work related to the use of deterministic importance functions for 
variance reduction of MC transport simulations is available in Reference 1.

This paper provides a review of the hybrid (Monte Carlo/deterministic) radiation transport methods and codes used at the Oak Ridge National Laboratory (ORNL) and examples of their application for increasing the efficiency of real-world, fixed-source MC simulations. These hybrid methods use approximate forward and/or adjoint fluxes from fast-running deterministic transport calculations to generate variance reduction parameters for accelerating fixed-source MC simulations.

\section{Methods}

The two principal hybrid methods used at ORNL are (1) Consistent Adjoint Driven Importance Sampling (CADIS) and (2) Forward Weighted CADIS (FW-CADIS). These methods were developed for optimizing fixed-source problems with different objectives, as summarized in Table 1, and are briefly reviewed in the following sections.

Table 1 Hybrid methods and uses

\begin{tabular}{ll}
\hline Hybrid method & \multicolumn{1}{c}{ Use } \\
\hline CADIS & $\begin{array}{l}\text { Optimization of localized detector } \\
\text { (tally) regions }\end{array}$ \\
\hline FW-CADIS & $\begin{array}{l}\text { Optimization of distributions (e.g., } \\
\text { mesh tallies) or multiple localized } \\
\text { detector regions }\end{array}$ \\
\hline
\end{tabular}

\section{Consistent Adjoint Driven Importance Sampling}

The CADIS method, ${ }^{1,2)}$ which is based on the recognition that the adjoint function (i.e., the solution to the adjoint Boltzmann transport equation) has physical significance as a measure of the importance of a particle to some objective function (e.g., the response of a detector), was originally developed at the Pennsylvania State University for the purpose of optimizing MC calculations of ex-core detector response in pressurized-water reactors (PWRs). ${ }^{3)}$ This method provides consistent formulations for source and transport biasing parameters and their implementation within the weight window technique.

The biased source distribution, $\hat{q}(\vec{r}, E)$, is given by the following relations:

$$
\begin{aligned}
& R=\iint q(\vec{r}, E) \phi^{+}(\vec{r}, E) d \vec{r} d E \\
& \hat{q}(\vec{r}, E)=\frac{\varphi^{+}(\vec{r}, E) q(\vec{r}, E)}{R}
\end{aligned}
$$

where $\phi^{+}(\vec{r}, E), q(\vec{r}, E)$, and $R$ are the scalar adjoint flux, the unbiased source, and the total detector response, respectively. By sampling from the biased source distribution, source particles are sampled in proportion to their expected contribution to the detector response.

For transport biasing, the weight window technique is employed. The weight-window technique provides a means for assigning detailed space- and energy-dependent impor- tances and applying geometric splitting/roulette and energy splitting/roulette, while at the same time controlling weight variations. The weight window technique has proven to be robust, reliable, and broadly applicable.

The weight-window technique requires weight-window lower bounds $w_{\ell}$. The width of the window is controlled by the input parameter $c$, which is the ratio of upper and lower weight-window bounds $\left(c=w_{u} / w_{\ell}\right)$. The space- and energy-dependent weight-window lower bounds are given by

$$
w_{l}(\vec{r}, E)=\frac{R}{\phi^{+}(\vec{r}, E)\left(\frac{c+1}{2}\right)}
$$

Note that because the source biasing parameters and weight-window lower bounds are consistent, the source particles are started with statistical weights $w_{0}$

$$
w_{0}(\vec{r}, E) \equiv \frac{q(\vec{r}, E)}{\hat{q}(\vec{r}, E)}=\frac{R}{\phi^{+}(\vec{r}, E)}
$$

that are within the weight windows, as desired. This is an important characteristic of the CADIS method because it eliminates the incompatibility between source and transport biasing that has been problematic in other approaches due to poor calculational efficiency and/or false convergence. ${ }^{4)}$ If the statistical weights of the source particles are not within the weight windows, the particles are immediately split or rouletted in an effort to bring their weights into the weight window. This results in unnecessary splitting/rouletting and a corresponding degradation in computational efficiency. Furthermore, for problems in which the adjoint function varies significantly within the source region (space and/or energy), the source biasing is very effective for improving computational efficiency.

As an illustration of CADIS, consider a spent fuel cask (2.3 $\mathrm{m}$ diameter, $4.8 \mathrm{~m}$ height) loaded with two different types of fuel, each of which contains activated hardware regions at the top and bottom of the assemblies. For this example, the goal is to calculate the total (neutron plus photon) dose rate at a specific location $1 \mathrm{~m}$ from the surface (a point detector).

To solve this problem with an analog calculation or with manual implementation of variance reduction, the first step a user must perform is to split the problem into separate simulations - one for the neutron sources and one for the photon sources - since the source strengths are so different in magnitude $\left(2.4 \times 10^{9}\right.$ neutron $/ \mathrm{s}$ and $7.2 \times 10^{16}$ photon/s) that analog sampling would not properly represent the neutron source. After $900 \mathrm{~min}$, the analog neutron calculation finds that the neutron dose rate is $3.84 \times 10^{-4} \mathrm{rem} / \mathrm{h}( \pm 3.0 \%)$ and the photon dose rate from neutron-gamma reactions is $3.02 \times 10^{-4} \mathrm{rem} / \mathrm{h}$ $( \pm 0.7 \%)$. These tallies passed the statistical tests. After 903 min, the analog photon calculation finds that the photon dose rate is $5.17 \times 10^{-3} \mathrm{rem} / \mathrm{h}( \pm 39.0 \%)$. This tally passed none of the statistical tests. This gives a total dose rate of $5.85 \times 10^{-3}$ $\mathrm{rem} / \mathrm{h}( \pm 34.4 \%)$. 
To solve this problem with an implementation of the CADIS method, all of the sources can be included together in one input, since the biased source will determine how to sample the various sources based on their contribution to the total dose at the detector. The original cask geometry, shown in Fig. 1, is voxelized into a discrete ordinates geometry $(67 \times 68 \times 69)$, also shown in Fig. 1 . The adjoint source is defined as a point source at the detector location, using the neutron and photon dose response functions as the energy spectrum. The resulting adjoint fluxes $\left(\mathrm{S}_{4}, \mathrm{P}_{1}\right)$, which took 6 min to calculate, are shown in Fig. 2. The adjoint fluxes are then used to create the biased sources, some of which are shown in Fig. 3, and the importance map target weight windows, as shown in Fig. 4. With a 24-min MC calculation, the dose rates computed by the single CADIS calculation are $3.74 \times 10^{-4} \mathrm{rem} / \mathrm{h}( \pm 1.5 \%)$ from neutrons, $8.01 \times 10^{-3} \mathrm{rem} / \mathrm{h}$ $( \pm 0.9 \%)$ from photons and $8.38 \times 10^{-3} \mathrm{rem} / \mathrm{h}( \pm 0.9 \%)$ total. These tallies satisfied all of the statistical checks. This is a factor of 93,000 improvement in figure-of-merit over the analog calculations.
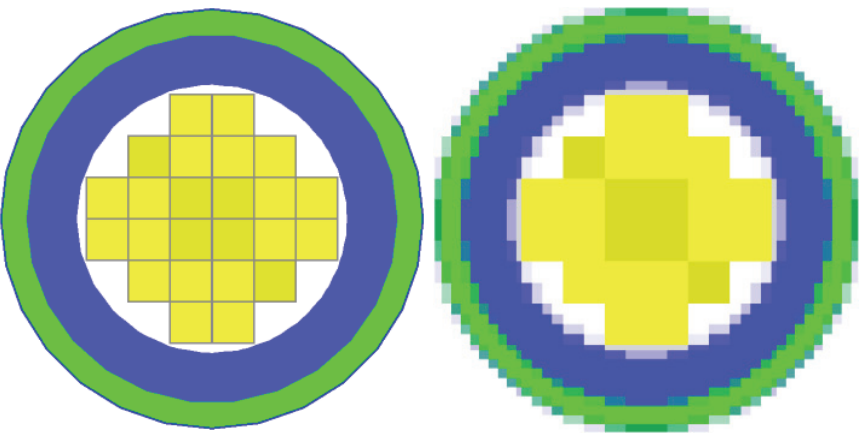

Fig. 1 The MC geometry (left) and the voxelized geometry (right). Note the two types of spent fuel-light and dark yellow.

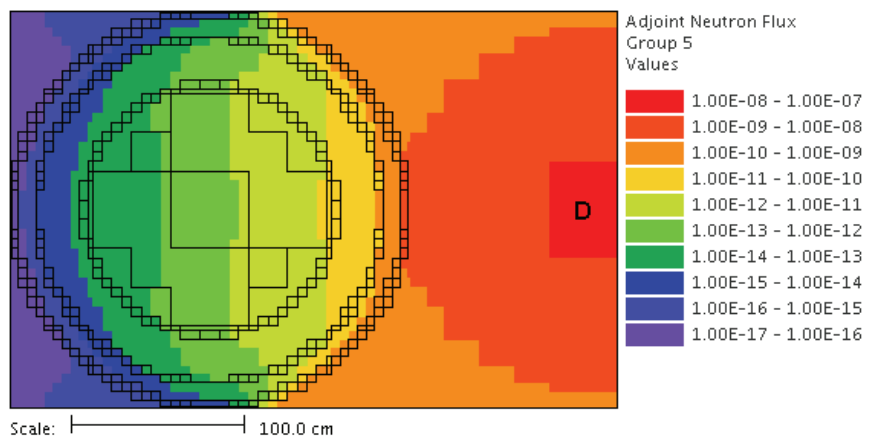

Fig. 2 The adjoint fluxes for $1-\mathrm{MeV}$ neutrons from an adjoint source at the detector position (D).

\section{Forward Weighted CADIS}

The FW-CADIS method ${ }^{5)}$ was originally conceived to simultaneously optimize near and far detectors in a nuclear well-logging tool $\left.{ }^{6}\right)$ but was not formalized and published until 2007. ${ }^{7)}$ Hence, in terms of publications, it is a relatively recent extension of the CADIS method that was developed for optimizing distributions (e.g., mesh tallies over all or part of the problem space) or multiple localized detector regions

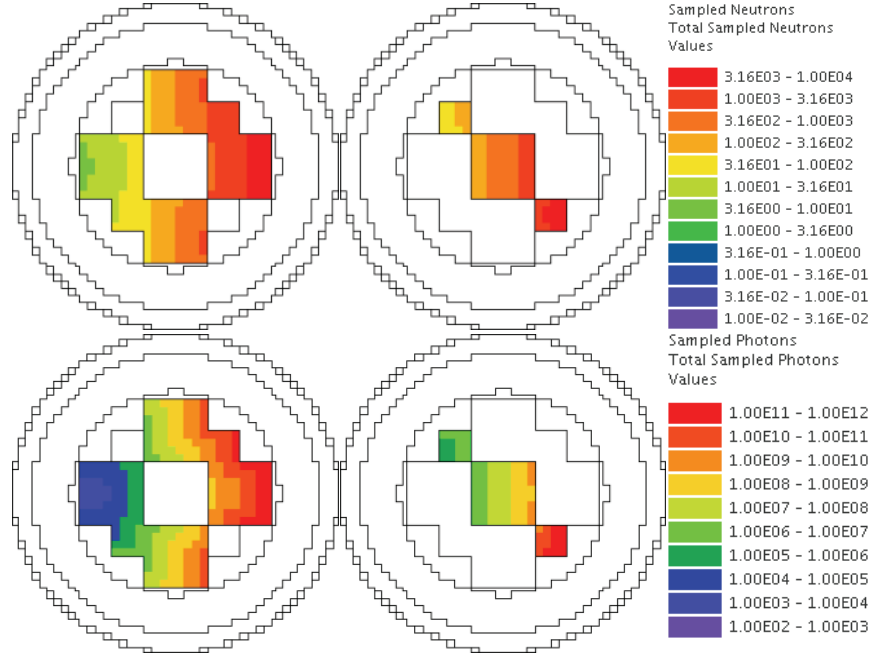

Fig. 3 Sampling distributions for four of the seven biased sources. The top row are neutron sources and the bottom row are photon sources. For every 12 fuel photons sampled, there is 1 neutron sampled.

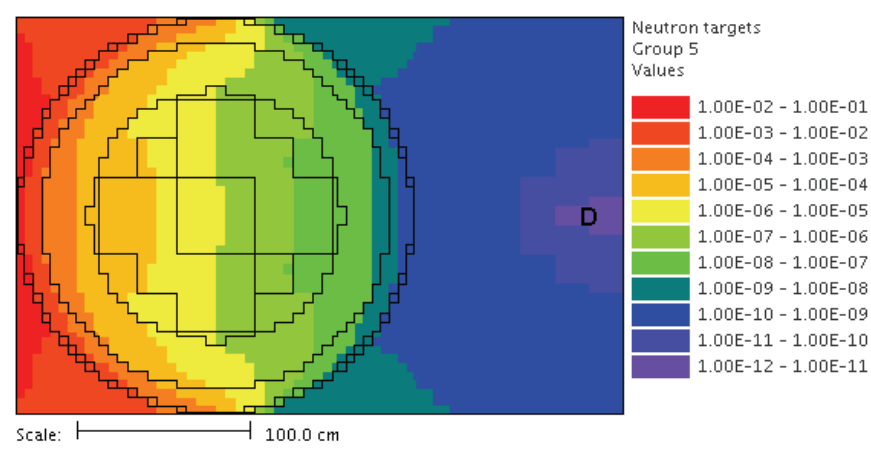

Fig. 4 The importance map target weights for $1-\mathrm{MeV}$ neutrons - particles moving towards lower weight areas are split, and particles moving toward higher weight areas are rouletted.

(e.g., simultaneous optimization of two or more localized tally regions).

The method involves determining a function that represents the importance of particles to achieving uniform MC particle density, which is related to statistical uncertainty, throughout all or part of the problem phase-space. The name stems from the fact that the method involves a weighting of the adjoint source $q^{+}(\vec{r}, E)$ with information from a forward solution $\phi(\vec{r}, E)$, i.e., forward weighting. For example, if the goal of a simulation is to calculate some response, such as dose rate, over a mesh tally using a detector response function, $\sigma_{d}(\vec{r}, E)$, then the adjoint source would be defined by

$$
q^{+}(\vec{r}, E)=\frac{\sigma_{d}(\vec{r}, E)}{\int \phi(\vec{r}, E) \sigma_{d}(\vec{r}, E) d E}
$$

The adjoint source is nonzero only where the mesh tally is defined and its magnitude is inversely proportional to the forward estimate of the response. Once the appropriate 
adjoint (importance) function is obtained, the standard CADIS relations for calculating consistent source biasing parameters and weight-window values are used. This method is significant in that it enables high-fidelity $\mathrm{MC}$ results for distributions (e.g., spatial dose distribution) in large phase spaces - a capability typically attributed only to deterministic methods.

As an example of FW-CADIS, consider the problem of finding dose rates everywhere in a PWR facility ${ }^{7)}$ from the operating reactor. Since reactor facilities are constructed to minimize dose to workers, this is a very challenging problem. An MCNP calculation without any variance reduction shows non-zero dose rates inside the reactor vessel and a few places inside containment that are not blocked by the steam generator shielding, as shown in Fig. 5. This calculation used nearly 10 billion histories and required 25 cpu $\bullet$ days to complete. Running the analog calculation out to $125 \mathrm{cpu} \bullet$ days does not increase the number of mesh tally voxels with non-zero scores.

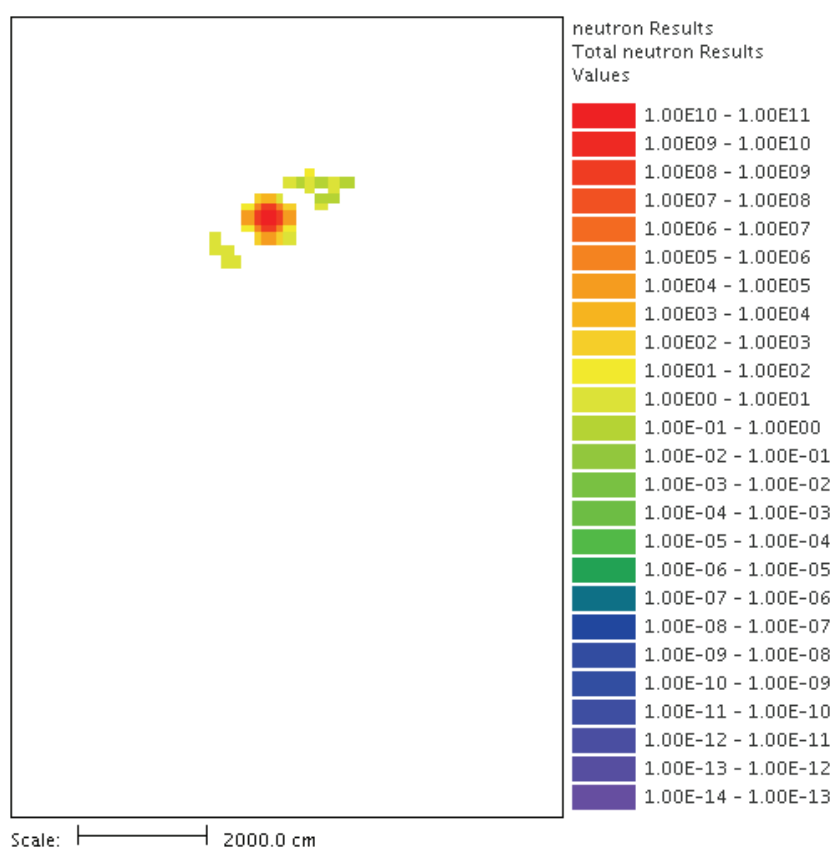

Fig. 525 -cpu•day analog calculation of neutron dose rate in a PWR facility.

The FW-CADIS calculation used a forward discrete ordinates calculation to estimate the neutron dose rates. The adjoint source was then defined as a box covering the entire facility and weighted inversely by the forward estimate of dose. Each discrete ordinates calculation required about 0.75 cpu•days. The adjoint fluxes were used to construct the biased source distribution and a weight-window input file for a 20 -cpu•days MC calculation. The resulting mesh tally is shown in Fig. 6. Note that the dose rates are non-zero over most of the facility and range over 30 orders of magnitude. In this case, the FW-CADIS enabled a calculation with MC that was otherwise not possible.

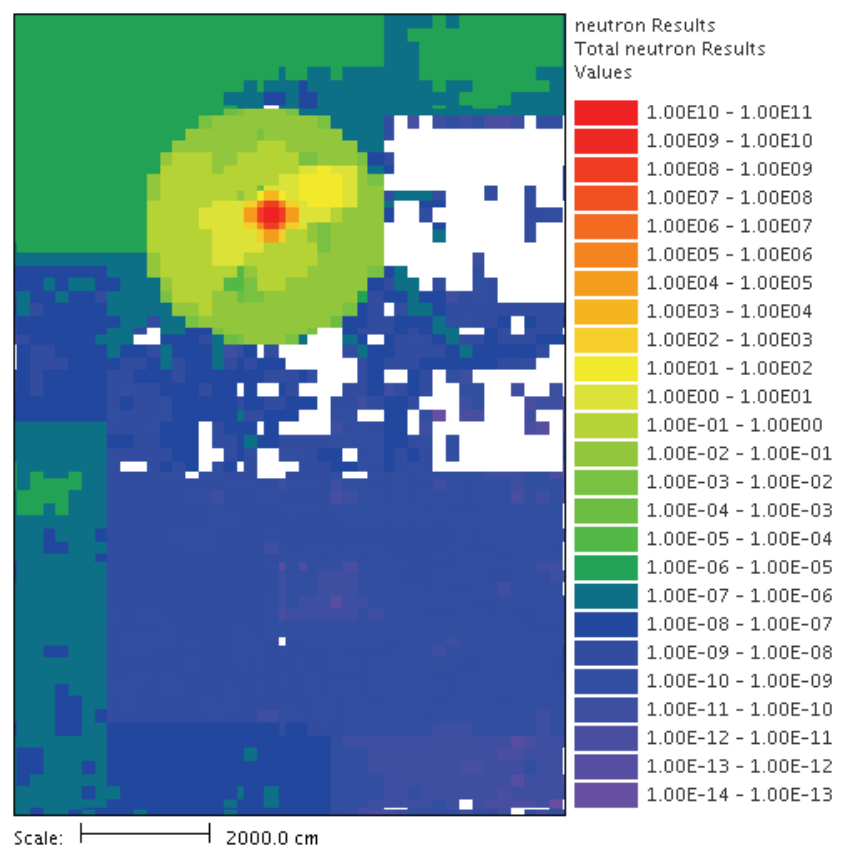

Fig. 6 FW-CADIS calculation of neutron dose rate in a PWR facility.

\section{Code Implementation}

The methods described above use approximate forward and/or adjoint fluxes from deterministic transport calculations to generate the variance reduction parameters needed to accelerate fixed-source MC simulations. Hence, both methods involve preparing input for deterministic calculations based on the MC input, performing deterministic calculations, and calculating variance reduction parameters based on the deterministic results. An important aspect relative to the usefulness of these methods is an implementation that minimizes user time, effort, and overall level of difficulty associated with completing the task. Hence, both methods have been implemented and automated in the SCALE MAVRIC sequence ${ }^{8)}$ (which uses the 3-D Monaco multi-group MC code) and the ADVANTG code ${ }^{9,10)}$ (which uses $\mathrm{MCNP}^{11)}$ for 3-D continuous-energy MC calculations). Both MAVRIC and ADVANTG use the Denovo 3-D discrete ordinates code for deterministic calculations. ${ }^{12,13)}$ Although the methods have been implemented to minimize the user effort, users must provide mesh boundaries and a description of the tallies to optimize.

The codes evaluate the geometry for each region using either a single material per cell or a homogenized material based on several test points within the cell ${ }^{14}$. The mesh needs to capture significant material changes and geometric details but does not need the fineness required of a stand-alone discrete ordinates calculation. Very fine meshing results in increased cost for the deterministic calculation and, in some cases, degraded performance in the MC calculation due to the size of the weight window map. The goal in the deterministic calculations is to quickly compute flux estimates - not to exactly solve the problem. 
The adjoint source corresponds to the tally or mesh area that the user wants to optimize, and the energy portion of the adjoint source is the response function for those tallies. FW-CADIS is used to compute several tallies with roughly the same uncertainty. This includes multiple tallies using the same response at different locations as well as different responses at different locations. Each tally becomes part of the adjoint source (spatial and energy), each divided by the forward estimate of the tally.

In ADVANTG, users also need to supply some information to relate the MCNP materials to the multi-group cross-section library used for the deterministic calculations. Based on the mesh, the adjoint source, and the materials information, MAVRIC and ADVANTG are able to prepare and run the required deterministic calculations and then generate the variance reduction parameters. Additional details on the implementation and user input for MAVRIC and ADVANTG are available in References 8 and 10 respectively.

\section{Applications}

\section{Consistent Adjoint Driven Importance Sampling}

The CADIS method has been used for more than a decade to dramatically improve the efficiency of MC simulations for a variety of neutron, gamma, and coupled neutron-gamma fixed-source source-detector-type problems. Examples of problems CADIS has been applied to and observed speed-ups are listed in Table 2. Generally speaking, the more difficult the tally, the larger the speed-up obtained via the CADIS method.

Table 2 Examples of applications and associated speed-ups for the CADIS method

\begin{tabular}{|c|c|c|}
\hline Application & $\begin{array}{c}\text { Observed speed-up } \\
\text { relative to manual } \\
\text { VR/analog } \\
\end{array}$ & Reference(s) \\
\hline $\begin{array}{l}\text { PWR cavity dosimetry } \\
\text { (high-energy reaction rates) }\end{array}$ & $4 / O\left(10^{4}\right)$ & $1-3$ \\
\hline DPA in BWR core shroud & $\mathrm{NA} / O\left(10^{3}\right)$ & 15 \\
\hline Neutron well-logging tool & $2-3 / O\left(10^{2}\right)$ & 6,16 \\
\hline Gamma well-logging tool & $\mathrm{NA} / O\left(10^{3-4}\right)$ & 6,16 \\
\hline $\begin{array}{l}\text { Dose from single spent fuel } \\
\text { storage cask }\end{array}$ & $\mathrm{NA} / O\left(10^{3-4}\right)$ & $1,17,18$ \\
\hline $\begin{array}{l}\text { PWR ex-core thermal de- } \\
\text { tector response }\end{array}$ & $\sim 13 / O\left(10^{4}\right)$ & 19 \\
\hline Passive threat detection & $\mathrm{NA} / O\left(10^{2-4}\right)$ & 20 \\
\hline $\begin{array}{l}\text { Active-interrogation SNM } \\
\text { detection }\end{array}$ & $\mathrm{NA} / O\left(10^{4}\right)$ & 20 \\
\hline ITER shielding & $\mathrm{NA} / O\left(10^{2-4}\right)$ & 21,22 \\
\hline HFIR DPA calculations & $\mathrm{NA} / O\left(10^{4}\right)$ & 23 \\
\hline $\begin{array}{l}\text { Criticality accident alarm } \\
\text { system analyses }\end{array}$ & $\mathrm{NA} / O\left(10^{2-3}\right)$ & 24,25 \\
\hline HTGR shielding analysis & NA / NA & 26 \\
\hline
\end{tabular}

\section{Forward Weighted CADIS}

The FW-CADIS method has been used for the past few years to dramatically improve the efficiency of MC simulations for a variety of neutron, gamma, and coupled neutron-gamma fixed-source problems in which results were sought throughout large portions of the problem space or at multiple detectors. In many cases, the method enabled MC solutions for problems that were otherwise not possible with MC. Examples of problems to which FW-CADIS has been applied are listed in Table 3. Note than in many cases it is either extremely difficult or impossible to obtain meaningful results with analog MC, and hence the speed-up is clearly very large but cannot be quantified.

Table 3 Examples of applications of the FW-CADIS method

\begin{tabular}{ll}
\hline \multicolumn{1}{c}{ Application } & Reference(s) \\
\hline Dose rate throughout full-scale PWR facility & $5,7,27$ \\
$\begin{array}{l}\text { Multiple detector responses in nuclear } \\
\text { well-logging tools }\end{array}$ & 6,16 \\
$\begin{array}{l}\text { Site boundary dose rate from an array of spent } \\
\text { fuel casks }\end{array}$ & 28,29 \\
$\begin{array}{l}\text { ITER shielding and material heating analysis } \\
\text { Dose rates throughout a critical facility }\end{array}$ & 21,22 \\
$\begin{array}{l}\text { Dose rates throughout an urban model from a } \\
\text { nuclear weapon detonation }\end{array}$ & 10 \\
\begin{tabular}{l} 
Dose rates throughout IRIS reactor containment \\
\hline
\end{tabular} & 30 \\
\hline
\end{tabular}

\section{Current Development Activities}

Development activities are ongoing at ORNL in the following areas:

1. Acceleration of reactor and criticality analyses - The FW-CADIS method has recently been extended and applied to eigenvalue problems for the purpose of accelerating the calculation of group-wise fluxes throughout a reactor core. ${ }^{31,32)}$ Current investigations include the use of deterministic importance functions to accelerate convergence of the fission source (eigenfunction), acceleration of the eigenvalue convergence, and acceleration of group-wise forward and adjoint fluxes for SCALE sensitivity/uncertainty analyses. ${ }^{8)}$

2. Acceleration for highly angular-dependent problems The CADIS implementation has recently been extended to provide angularly-dependent weight windows for applications including active-interrogation problems and beamline shielding analyses for the ORNL Spallation Neutron Source. ${ }^{33,34)}$ Using weight-window targets with the full angular information from the adjoint discrete ordinates calculation requires an enormous amount of memory. Hence, formalisms using a $\mathrm{P}_{1}$-type approximation, similar to previous angular weight-window schemes, ${ }^{35)}$ have been developed and combined with a consistent biased source distribution. For many of the 
applications in this paper, speed-ups of a factor of two to three were observed with the space/energy/angle CADIS, as compared to the standard space/energy CADIS.

3. Acceleration of active-interrogation problems - A new, multi-step CADIS-based approach has been developed and implemented to address the challenging aspects of simulating active interrogation techniques for locating special nuclear material (SNM) in large cargo containers. ${ }^{36,37)}$ The multi-step approach involves optimizing the interaction (fission) rate in the SNM from the interrogating (source) particles, followed by optimizing the detector response from interaction progeny of interest.

4. Improvement of use - New features designed to improve reliability and robustness and reduce user requirements have been developed and implemented. ${ }^{38)}$ Efforts continue in this area related to more intelligent automated meshing for the deterministic calculations and improved diagnostics and automated mitigation of poor statistical convergence behavior. ${ }^{39)}$

\section{Conclusion}

This paper provides an overview of the hybrid (Monte Carlo/deterministic) radiation transport methods and codes developed and used at ORNL and specific examples of their application for dramatically increasing the efficiency of real-world, fixed-source Monte Carlo analyses. While substantial speed-ups $\left[O\left(10^{2-4}\right)\right]$ are routinely observed with the CADIS method, the FW-CADIS method has proven to be a transformational method in that it enables high-fidelity $\mathrm{MC}$ results for distributions (e.g., spatial dose distribution) in large phase spaces - a capability previously attributed only to deterministic methods. Hence, this method has initiated a paradigm shift in the methodology used to obtain accurate, full domain solutions to large, complex radiation transport problems.

\section{Acknowledgment}

This review paper was sponsored by the Laboratory Directed Research and Development program at the Oak Ridge National Laboratory, managed by UT-Battelle, LLC for the U.S. Department of Energy.

\section{References}

1) A. Haghighat, J. C. Wagner, "Monte Carlo Variance Reduction with Deterministic Importance Functions," Prog. Nucl. Energy, 42[1], 25-53 (2003).

2) J. C. Wagner, A. Haghighat, "Automated Variance Reduction of Monte Carlo Shielding Calculations Using the Discrete Ordinates Adjoint Function," Nucl. Sci. Eng., 128, 186 (1998).

3) J. C. Wagner, Acceleration of Monte Carlo Shielding Calculations with an Automated Variance Reduction Technique and Parallel Processing, Ph.D. Dissertation, Pennsylvania State University, University Park, Pennsylvania (1997).

4) J. S. Hendricks, C. N. Culbertson, "An Assessment of MCNP Weight Windows," Proc. of the PHYSOR 2000 ANS International Topical Meeting on Advances in Reactor Physics and Mathematics and Computation into the Next Millennium, Pittsburgh, PA, USA, May 7-12, 2000 (2000).
5) J. C. Wagner, E. D. Blakeman, D. E. Peplow, "Forward-Weighted CADIS Method for Variance Reduction of Monte Carlo Calculations of Distributions and Multiple Localized Quantities," M\&C 2009, Saratoga Springs, N.Y., May 3-7, 2009 (2009).

6) J. C. Wagner, "An Automated Deterministic Variance Reduction Generator for Monte Carlo Shielding Applications," Presentation at the ANS 12th Biennial Topical Meeting of the Radiation Protection and Shielding Division, Santa Fe, New Mexico, April 14-18, 2002 (2002).

7) J. C. Wagner, E. D. Blakeman, D. E. Peplow, "Forward-Weighted CADIS Method for Global Variance Reduction," Trans. Am. Nucl. Soc., 97, 630-633 (2007).

8) SCALE: A Modular Code System for Performing Standardized Computer Analyses for Licensing Evaluation, ORNL/TM-2005/39, Version 6, Vols. I-III. Available from Radiation Safety Information Computational Center at Oak Ridge National Laboratory as CCC-750 (2009).

9) J. C. Wagner, "An Automated Deterministic Variance Reduction Generator for Monte Carlo Shielding Applications," Proc. ANS 12th Biennial Topical Meeting of the Radiation Protection and Shielding Division, Santa Fe, New Mexico, April 14-18, $2002(2002)$.

10) S. W. Mosher, "A New Version of the ADVANTG Variance Reduction Generator," American Nuclear Society Radiation Protection and Shielding Division 2010 Topical Meeting, Las Vegas, Nevada, April 18-23, 2010 (2010).

11) X-5 MONTE CARLO TEAM, MCNP-A General Monte Carlo N-Particle Transport Code, Version 5. Volume I: Overview and Theory, LA-UR-03-1987, Los Alamos National Laboratory (LANL) (2003).

12) T. M. Evans, A. S. Stafford, K. T. Clarno, "Denovo-A New Three-Dimensional Parallel Discrete Ordinates Code in SCALE," Nucl. Technol., 171, 171-200 (2010).

13) T. M. Evans, G. G. Davidson, R. N. Slaybaugh, "Three-Dimensional Full Core Power Calculations for Pressurized Water Reactors," SCIDAC 2010, Chattanooga, Tennessee, July 11-15, 2010 (2010).

14) A. M. Ibrahim, D. E. Peplow, T. M. Evans, J. C. Wagner, P. P. H. Wilson, "Improving the Mesh Generation Capabilities in the SCALE Hybrid Shielding Analysis Sequence," Trans. Am. Nucl. Soc., 100, 302-304 (2009).

15) A. Haghighat, H. Hiruta, B. Petrovic, J. C. Wagner, "Performance of the Automated Adjoint Accelerated MCNP (A $\left.{ }^{3} \mathrm{MCNP}\right)$ for Simulation of a BWR Core Shroud Problem," Proc. Int. Conf. on Mathematics and Computation, Reactor Physics, and Environmental Analysis in Nuclear Applications, Madrid, Spain, September 27-30, 1999 (1999).

16) J. C. Wagner, D. E. Peplow, T.M. Evans, "Automated Variance Reduction Applied to Nuclear Well-Logging Problems," Nucl. Technol., 168, 799-809 (2009).

17) D. E. Peplow, J. C. Wagner, "Automated Variance Reduction for SCALE Shielding Calculations," Proc. ANS 14th Biennial Topical Meeting of the Radiation Protection and Shielding Division, Carlsbad, New Mexico, April 2-6, 2006, pp. 556-558 (2006).

18) D. E. Peplow, "Monte Carlo Shielding Analysis Capabilities with MAVRIC," accepted for publication by Nucl. Technol. (2011).

19) H. P. Smith, J. C. Wagner, "A Case Study in Manual and Automated Monte Carlo Variance Reduction with a Deep Penetration Reactor Shielding Problem," Nucl. Sci. Eng., 149, 23-37 (2005).

20) S. W. Mosher, T. M. Miller, T. M. Evans, J. C. Wagner, “Automated Weight-Window Generation for Threat Detection 
Applications using ADVANTG," M\&C 2009, Saratoga Springs, N.Y., May 3-7, 2009 (2009).

21) A. M. Ibrahim, S. W. Mosher, T. M. Evans, D. E. Peplow, M. E. Sawan, P. P. H. Wilson, J. C. Wagner, "ITER Neutronics Modeling Using Hybrid Monte Carlo/Deterministic and CAD-based Monte Carlo Methods," accepted for publication Nucl. Technol.

22) A. M. Ibrahim, S. W. Mosher, T. M. Evans, D. E. Peplow, M. E. Sawan, P. P. H. Wilson, J. C. Wagner, "ITER Neutronics Modeling Using Hybrid Monte Carlo/ $\mathrm{S}_{\mathrm{N}}$ and CAD-Based Monte Carlo Methods," American Nuclear Society Radiation Protection and Shielding Division 2010 Topical Meeting, Las Vegas, Nevada, April 18-23, 2010 (2010).

23) E. D. Blakeman, "HFIR dpa-Rate Calculations Using Hybrid Monte Carlo/Discrete Ordinates," American Nuclear Society Radiation Protection and Shielding Division 2010 Topical Meeting, Las Vegas, Nevada, April 18-23, 2010 (2010).

24) D. E. Peplow, L. M. Petrie, Jr., "Criticality Accident Alarm System Modeling with SCALE," M\&C 2009, Saratoga Springs, N.Y., May 3-7, 2009 (2009).

25) D. E. Peplow, L. M. Petrie, Jr., "Criticality Accident Alarm System Modeling Made Easy With SCALE 6.1," Trans. Am. Nucl. Soc., 102, 297-299 (2010).

26) E. D. Blakeman, Modeling and Analysis of the ORNL HTGR Neutron-Streaming Experiment using SCALE/MAVRIC, in progress, ORNL/TM-2010/196, Oak Ridge National Laboratory (ORNL) (2010).

27) E. D. Blakeman, D. E. Peplow, J. C. Wagner, B. D. Murphy, D. E. Mueller, PWR Facility Dose Modeling Using MCNP5 and the CADIS/ADVANTG Variance-Reduction Methodology, ORNL/TM-2007/133, Oak Ridge National Laboratory (ORNL) (2007).

28) D. E. Peplow, E. D. Blakeman, J. C. Wagner, “Advanced Variance Reduction Strategies for Optimizing Mesh Tallies in MAVRIC," Trans. Am. Nucl. Soc. 97, 595-597 (2007).

29) D. E. Peplow, T. M. Evans, J. C. Wagner, "Simultaneous Optimization of Tallies in Difficult Shielding Problems," Nucl. Technol., 168, 785-792 (2009).

30) B. Petrovic, "Preliminary Evaluation of the SCALE MAVRIC Code and FW-CADIS Method for Efficient Scoping of the Radiation Field Throughout the IRIS Containment," Nucl. Technol., 168, 438-443 (2009).
31) J. C. Wagner, S. W. Mosher, "Forward-Weighted CADIS Method for Variance Reduction of Monte Carlo Reactor Analyses," Trans. Am. Nucl. Soc., 103, 342-345 (2010).

32) J. C. Wagner, T. M. Evans, S. W. Mosher, D. E. Peplow, J. A. Turner, "Hybrid and Parallel Domain-Decomposition Methods Development to Enable Monte Carlo for Reactor Analyses," Joint Int. Conf. on Supercomputing in Nuclear Applications + Monte Carlo 2010, Tokyo, Japan (2010).

33) D. E. Peplow, S. W. Mosher, T. M. Evans, "Hybrid Monte Carlo/Deterministic Methods for Streaming/Beam Problems," American Nuclear Society Radiation Protection and Shielding Division 2010 Topical Meeting, Las Vegas, Nevada, April 18-23 (2010).

34) D. E. Peplow, S. W. Mosher, T. M. Evans, J. C. Wagner, "Hybrid Monte Carlo/Deterministic Methods for Streaming/Beam Problems," in progress for Nucl. Technol. (2011).

35) K. A. Van Riper, T. J. Urbatsch, P. D. Soran, D. K. Parsons, J. E. Morel, G. W. McKinney, S. R. Lee, L. A. Crotzer, F. W. Brinkley, J. W. Anderson, R. E. Alcouffe, "AVATAR-Automatic Variance Reduction in Monte Carlo Calculations," Proc. Joint Int. Conf. on Mathematical Methods and Supercomputing in Nuclear Applications, Saratoga Springs, New York, October 6-10, 1997, Vol.1, p.661 (1997).

36) D. E. Peplow, T. M. Miller, B. W. Patton, "Hybrid Monte Carlo/Deterministic Methods for Active Interrogation Modeling," American Nuclear Society Radiation Protection and Shielding Division 2010 Topical Meeting, Las Vegas, Nev., April 18-23 (2010).

37) D. E. Peplow, T. M. Miller, B. W. Patton, J. C. Wagner, "Hybrid Monte Carlo/Deterministic Methods for Active Interrogation Modeling," in progress for Nucl. Technol. (2011).

38) A. Ibrahim, D. E. Peplow, T. M. Evans, P. P. H. Wilson, J. C. Wagner, "Improving the $\mathrm{S}_{\mathrm{N}}$ Adjoint Source and Geometry Representation Capabilities in the SCALE Hybrid Shielding Analysis Sequence," American Nuclear Society Radiation Protection and Shielding Division 2010 Topical Meeting, Las Vegas, Nev., April 18-23 (2010).

39) A. Ibrahim, "Automatic Mesh Adaptivity for the Hybrid Monte Carlo/Deterministic Techniques of Neutronics Shielding Simulations," in progress for Nucl. Sci. Eng. 